\title{
Impact of a restrictive antibiotic policy on the acquisition of extended-spectrum beta-lactamase- producing Enterobacteriaceae in an endemic region: a quasi-experimental, before-and-after, propensity- matched cohort study in a Caribbean intensive care unit
}

Christophe Le Terrier ( $\boldsymbol{\sim}$ leterrier.icu@gmail.com )

University Hospitals Geneva: Hopitaux Universitaires Geneve https://orcid.org/0000-0002-5455-5576

Marco Vinetti

Hospital Saint-Pierre Ottignies: Clinique Saint-Pierre Ottignies

Paul Bonjean

CHU Saint-Étienne: Centre Hospitalier Universitaire de Saint-Etienne

Régine Richard

University Hospital Centre Pointe-a-Pitre Abymes: Centre Hospitalier Universitaire Pointe-a-Pitre Abymes

Bruno Jarrige

University Hospital Centre Pointe-a-Pitre Abymes: Centre Hospitalier Universitaire Pointe-a-Pitre Abymes

\section{Bertrand Pons}

University Hospital Centre Pointe-a-Pitre Abymes: Centre Hospitalier Universitaire Pointe-a-Pitre Abymes

\section{Benjamin Madeux}

University Hospital Centre Pointe-a-Pitre Abymes: Centre Hospitalier Universitaire Pointe-a-Pitre Abymes

\section{Pascale Piednoir}

University Hospital Centre Pointe-a-Pitre Abymes: Centre Hospitalier Universitaire Pointe-a-Pitre Abymes

\section{Fanny Ardisson}

University Hospital Centre Pointe-a-Pitre Abymes: Centre Hospitalier Universitaire Pointe-a-Pitre Abymes Elain Elie

University Hospital Centre Pointe-a-Pitre Abymes: Centre Hospitalier Universitaire Pointe-a-Pitre Abymes Frédéric Martino

University Hospital Centre Pointe-a-Pitre Abymes: Centre Hospitalier Universitaire Pointe-a-Pitre Abymes Marc Valette

University Hospital Centre Pointe-a-Pitre Abymes: Centre Hospitalier Universitaire Pointe-a-Pitre Abymes Édouard Ollier

University Hospital Centre Saint-Étienne: Centre Hospitalier Universitaire de Saint-Etienne

\section{Sébastien Breurec}


University Hospital Centre Pointe-a-Pitre Abymes: Centre Hospitalier Universitaire Pointe-a-Pitre Abymes

\section{Michel Carles}

CHU Nice: Centre Hospitalier Universitaire de Nice

\section{Guillaume Thiéry}

University Hospital Centre Saint-Étienne: Centre Hospitalier Universitaire de Saint-Etienne

\section{Research}

Keywords: intensive care unit, Caribbean, extended-spectrum beta-lactamase (ESBL)-producing Enterobacteriaceae, antimicrobial resistance, antibiotic stewardship, Intestinal microbiota, ESBL-E colonization

Posted Date: May 6th, 2021

DOl: https://doi.org/10.21203/rs.3.rs-480367/v1

License: (c) (1) This work is licensed under a Creative Commons Attribution 4.0 International License. Read Full License

Version of Record: A version of this preprint was published at Critical Care on July 26th, 2021. See the published version at https://doi.org/10.1186/s13054-021-03660-z. 


\section{Abstract}

Background: High-level antibiotic consumption plays a critical role in the selection and spread of extended-spectrum beta-lactamase-producing Enterobacteriaceae (ESBL-E) in ICU. Implementation of a stewardship program including a restrictive antibiotic policy was evaluated with respect to the ESBL-E acquisition (carriage and infection).

Methods: We implemented a two-years, quasi-experimental, before-and-after intervention study with all consecutive adult patients admitted for $>48 \mathrm{~h}$ in the medical-surgical 26-bed ICU of Guadeloupe University Hospital (French West Indies). A conventional strategy period (CSP), including a broadspectrum antibiotic as initial empirical treatment, followed by de-escalation (period before) was compared to a restrictive strategy period (RSP) limiting broad-spectrum antibiotics and shortening their duration. Antibiotic therapy was delayed and initiated only after microbiological identification, except for septic shock, severe acute respiratory distress syndrome and meningitis (period after). A multivariate Cox proportional hazard regression model adjusted on propensity score values was performed. The main outcome was the median time of being free of ESBL-E in the ICU. Secondary outcome included all-cause ICU mortality.

Results: The study included 1,541 patients, for a total of 738 in the CSP and 803 in the RSP. During the RSP, less patients were treated with antibiotics ( $46.8 \%$ vs $57.9 \% ; p<0.01)$, treatment duration was shorter ( 5 vs 6 days; $p<0.01$ ), administration of antibiotics targeting anaerobic pathogens significantly decreased (65.3\% vs 33.5\%; $p<0.01)$ compared to the CSP. The incidence of ICU-acquired ESBL-E was lower $(12.1 \%$ vs $19 \% ; p<0.01$ ) during the RSP. The median time of being free of ESBL-E was 22 days ( $95 \%$ confidence interval, 16-NA days) in the RSP and 18 days ( $95 \% \mathrm{Cl}, 16-21$ days) in the CSP. After propensity score weighting and adjusted analysis, the median time of being free of ESBL-E was independently associated with the RSP (hazard ratio, 0.746 [95\% $\mathrm{Cl}, 0.575-0.968$ ]; $p=0.02$, and hazard ratio 0.751 [95\% $\mathrm{Cl} 0.578$ 0.977 ]; $p=0.03$, respectively). All-cause ICU mortality were lower in the RSP than in the CSP $(22.5 \%$ vs $28.6 \% ; p<0.01)$.

Conclusions: Implementation of a program including a restrictive antibiotic strategy is feasible and is associated with less ESBL-E acquisition in the ICU without any worsening of patient outcome.

\section{Introduction}

Antibiotic resistance is among the most important public health concerns worldwide (1). Recently, the World Health Organization published a global priority list of antibiotic-resistant bacteria in which extended-spectrum beta-lactamase-producing Enterobacteriaceae (ESBL-E) are included in the priority 1 group (2). In intensive care units (ICUs), ESBL-E have been increasingly reported for many years, which strengthens the requirement for efficient prevention strategies (3). Occurrence of ESBL-E in ICU may result either from the introduction of an exogenous strain through newly-hospitalized patients with a possible further dissemination through cross-contamination, or from the in-vivo selection of resistant isolates from 
pre-existing strains, mainly in the gut microbiota, through horizontal gene transfer (4). Among risk factors for ESBL-E acquisition, antibiotic exposure to broad-spectrum cephalosporins and beta-lactam/betalactamase inhibitor combinations has been identified as an independent risk factor for colonization or infection with ESBL-E pathogens (5).

Antibiotic therapy is heavily used in ICUs where it has been reported that more than $70 \%$ of patients are treated with at least one antibiotic (6). Consequently, antibiotic overuse and the resulting selection pressure makes the ICU an important determinant of the spread of ESBL-E in the hospital $(7,8)$. Different stewardship policies have been developed and implemented in many settings, including ICUs, to improve antibiotic use and clinical outcomes, and to reduce the overall antibiotic selective pressure (7-9). Among those strategies that have been implemented to optimize antibiotic prescription in ICUs, some restrictive policies, such as delaying the initiation of antibiotics in selected patients or avoiding broad-spectrum antibiotic therapy, have successfully been proposed (10).

In the Caribbean region, the prevalence of multidrug-resistant bacteria is high, including $\operatorname{ESBL-E}(11,12)$, and this particular local ecology often leads clinicians to use broad-spectrum antibiotics empirically (13). Although stewardship programs are urgently needed, no such restrictive strategy has been evaluated in ICUs. In order to overcome this issue, we implemented a stewardship program based on a restrictive antibiotic policy. The aim of this study was to evaluate the impact this strategy on ESBL-E acquisition in the ICU, compared to a conventional and unrestricted antibiotic policy.

\section{Methods}

\section{Study Design and Setting}

We conducted a retrospective, observational, quasi-experimental, before-and-after intervention study from 1 January 2014 to 31 December 2015 in a 26-bed ICU admitting medical and surgical patients at Guadeloupe University Hospital (French West Indies). The ethics committee of the French Society of Intensive Care Medicine (CE SRLF 18-44) approved the study and granted a waiver for informed consent because that both treatment methods were classified as standard care. This manuscript follows the STROBE statement for reporting of cohort studies.

Rectal swabs (ESBL-E screening) were performed at ICU admission and once-weekly until discharge, as well as upon admission to the next unit. In that latter case, a positivity in ESBL-E carriage was attributed to the ICU. Contact isolation precautions were applied for each patient until the first swab results were obtained. Alcohol-based handrub was routinely used for hand hygiene. None of these procedures was modified during the study period. All patients admitted to the ICU $>48 \mathrm{~h}$ during the study period were included in the analysis and followed up until hospital discharge or death. Patients for whom ESBL-E carriage was unknown on ICU admission were not included.

The outcome of interest was the median time of being free of ESBL-E in the ICU, defined by the time to acquire an ESBL-E in a competing event of death during the follow-up. Secondary outcomes were the 
incidence of ICU-acquired ESBL-E, duration of antibiotic therapy, antibiotic-free days until ICU discharge, all-cause hospital and ICU mortality, ICU and hospital length of stay, ICU-acquired infections and bacteremia with ESBL-E, and relapse or recurrence of sepsis. A subgroup of ICU patients receiving antibiotic therapy was also analyzed to investigate outcomes in those directly exposed by the restrictive antibiotic stewardship strategy.

\section{Procedures}

The 2-year study period was split into two 1-year periods, which differed by the antibiotic policy employed. During the first year, the "conventional strategy period" (CSP), antibiotic therapy was prescribed at the physician's discretion based on national and international guidelines. This strategy included the use of a broad-spectrum antibiotic as initial empirical treatment in the case of sepsis or suspected infection, followed by de-escalation after 48 to $72 \mathrm{~h}$, based on microbiological data. The main regimens were combination therapies with a cephalosporin and aminoglycoside for community-acquired infections, and carbapenem or piperacillin/tazobactam combined with amikacin for hospital-acquired infections. Dosage, timing and duration followed French guidelines (14).

As part of a stewardship program, a new set of guidelines with a very restrictive antibiotic protocol was established by the ICU team, approved by a multidisciplinary team and implemented on 1 January 2015. The "restrictive strategy period" (RSP) was based on seven principles. 1) For suspected infection, microbiological samples were taken immediately, and antibiotic therapy was initiated only after microbiological identification, except for septic shock, severe acute respiratory distress syndrome (ARDS) and meningitis. 2) For non-documented septic shock and severe ARDS, an empiric combination therapy including a cephalosporin and an aminoglycoside was immediately started after microbiological sampling according to the ICU protocol. Combined therapy included either second or third-cephalosporin (cefuroxime, cefotaxime or ceftriaxone) for community-acquired septic shock, or cefoxitin for hospitalacquired septic shock (owing the resistance to the previously-listed cephalosporins and the high rate of susceptibility to cefoxitin of the ESBL-E) or an anti Pseudomonas aeruginosa cephalosporin (ceftazidime or cefepime) for late ( $>5$ days) ventilation-acquired pneumonia (VAP). The second antibiotic was amikacin, unless a Gram-positive pathogen was highly suspected. Due to the very low prevalence of methicillin-resistant Staphylococcus aureus (MRSA) in our hospital, the first-line anti-staphylococcal treatment was cefazolin. 3) No use of piperacillin/tazobactam and carbapenems for empirical treatment, only for a documented infection without an alternative option. 4) Limited coverage on $P$. aeruginosa, unless clearly indicated. 5) Limited coverage on subdiaphragmatic anaerobes, unless clearly indicated. 6) Monotherapy as a definitive treatment. 7) Other characteristics of antibiotic treatment were short duration, high doses, and de-escalation as soon as possible to the narrowest alternative (15), with a focus on penicillin, first- and second-generation cephalosporins, according to the attending physician and following ICU protocols (see detailed protocol in Additional file 1). 


\section{Data Collection}

Clinical and laboratory findings were collected from the patient's medical records. In the CSP, diagnosis of infection and sepsis was based on the clinical judgment of the attending physician. During the RSP, the diagnosis required the identification of a pathogen and/or a source of infection. Septic shock was defined by an infection associated with the need of vasoactive drugs.

ESBL-E carriage on ICU admission was defined by a positive rectal swab or positive culture without evidence of clinical infection. ICU-acquired ESBL-E was defined by a positive swab or positive culture $48 \mathrm{~h}$ or more after a negative culture at admission. ESBL-E infection was defined as a positive culture with evidence of clinical infection and detected by chromID ESBL ${ }^{\circledR}$ (bioMérieux, Marcy l'Etoile, France), a ready-to-use chromogenic selective medium for ESBL-producing Enterobacteriaceae. Antibiotic susceptibility was tested by the disk diffusion method on Mueller-Hinton agar (Bio-Rad, Hercules, CA, USA), and production of ESBL was confirmed by the double-disk synergy test according to the guidelines of the European Committee on Antimicrobial Susceptibility Testing (16). Due to the very low incidence in our hospital, Carbapenemase-producing Enterobacteriaceae were not analyzed in this study. VAP was diagnosed using standard criteria (17). Diagnosis of VAP required microbiological confirmation by quantitative culture of a common pathogens. Other ICU-acquired infections were diagnosed using standard criteria, with microbiological documentation for all cases.

\section{Statistical Analysis}

Data are reported as frequencies and proportions for categorical variables and mean, median, standard deviation, and $1 \mathrm{st}$ and 3rd quartiles for continuous variables. Patients hospitalized in the CSP were compared with those hospitalized in the RSP using the chi-square or Fisher's tests for categorical variables and Wilcoxon-Mann-Whitney tests for continuous variables. To assess the relationship between the strategy period and the median time of being free of ESBL-E, we calculated the Kaplan-Meier curve and used a univariate Cox proportional hazard regression model to estimate the hazard ratio (HR) and its $95 \%$ confidence interval. The follow-up time used for survival analyses corresponds to the time between ICU admission and ESBL-E acquisition or ICU discharge from intensive care (or death) if no ESBL-E acquisition occurring during the ICU stay.

To balance confounding factors, a propensity score to receive the restrictive strategy was calculated using a logistic regression model including either clinically relevant or statistically significant covariates (age, Simplified Acute Physiology Score [SAPS] II, chronic renal failure, diabetes, immunosuppression, antibiotherapy prior to admission, sepsis [at admission or occurring during ICU stay], other multidrug bacteria carriage at admission, and length of ventilation $>48$ hours) (Figure 1, Additional file 1). Fifty-two patients were excluded due to missing values on relevant variables. A comparison between excluded and included patients showed a significantly superior number of included patients with less than 48 hours of ventilation $(52 \%$ vs $17.3 \% ; p<0.001)$ and sepsis at admission $(33.2 \%$ vs $5.8 \% ; p<0.001)$. The area under 
the ROC curve estimating the predictive score ability was 0.612 (95\% Cl, 0.609-0.614) (Figure 2, Additional file 1). In the weighted dataset, all absolute standardized differences were inferior to $5 \%$, thus reflecting the good comparability of both groups. This score was used to calculate the inverse probability of treatment weights, assigning patients receiving a restrictive strategy a weight of $1 \div$ (propensity score) and those receiving a conventional strategy a weight of $1 \div(1$ - propensity score), with the use of stabilized weights to reduce variability. Balance among covariates was assessed in the weighted dataset using absolute standardized differences and all results were inferior to $5 \%$. These weights were then used to estimate the relationship between the strategy used and the median time of being free of ESBL-E in a univariate Cox proportional hazard regression weighted model. Kaplan-Meier curves of both groups from the weighted dataset were also estimated. A sensitivity analysis was performed by estimating the impact of the intervention using a multivariate Cox proportional hazard regression model adjusted on the propensity score values. All tests were conducted at a two-sided alpha risk of $5 \%$. Analyses were performed using the 4.0.3 version of $R$.

\section{Results}

\section{Patient Characteristics and Outcomes}

We included 1,541 patients in the study (CSP: 738; RSP; 803) (Figure 1). Baseline characteristics are summarized in Table 1. RSP patients had a lower SAPS II, less diagnosis of sepsis on admission. Less patients in the RSP presented with sepsis or septic shock (40.7\% vs $51.5 \% ; p<0.01)$, but there was no statistical difference in the proportion of patients receiving vasoactive drugs for septic shock during the two periods ( $20.3 \%$ vs $24.3 \%$; $p=0.06$ ). Similar ICU mortality was observed in patients admitted for a length of stay $<48$ hours as safety criteria. No difference in a specific sepsis category was reported at ICU admission. However, more pulmonary infections and less intra-abdominal infections were diagnosed during ICU stay in the CSP group (Table 1, Additional file 1).

Table 1. Demographic characteristics, comorbidities and diagnosis upon admission to the ICU of all included patients. 


\begin{tabular}{|c|c|c|c|}
\hline \multirow[t]{3}{*}{ Patients' characteristics } & $\begin{array}{l}\text { Conventional strategy } \\
\text { period }\end{array}$ & $\begin{array}{l}\text { Restrictive strategy } \\
\text { period }\end{array}$ & $\begin{array}{l}p \\
\text { value }\end{array}$ \\
\hline & 2014 & 2015 & \\
\hline & $n=738$ & $n=803$ & \\
\hline Age, years, median (IQR) & $59(45-69)$ & $58(42-70)$ & 0.53 \\
\hline Men (\%) & 64.0 & 63.1 & 0.74 \\
\hline SAPS II, median (IQR) & $40(26-55)$ & $37(24-53)$ & $<0.01$ \\
\hline \multicolumn{4}{|l|}{$\begin{array}{l}\text { Comorbidities before ICU admission } \\
n(\%)\end{array}$} \\
\hline Hypertension & $284(38.5)$ & $349(43.5)$ & 0.05 \\
\hline Diabetes & $220(29.8)$ & $207(25.8)$ & $<0.01$ \\
\hline Chronic renal insufficiency & $78(10.6)$ & $102(12.7)$ & 0.19 \\
\hline Cardiac disease & $73(9.9)$ & $67(8.3)$ & 0.29 \\
\hline Immunosuppression & $48(6.5)$ & $89(11.1)$ & $<0.01$ \\
\hline Malignancy & $67(9.1)$ & $62(7.7)$ & 0.34 \\
\hline Obesity ${ }^{a}$ & $52(7.0)$ & $65(8.1)$ & 0.44 \\
\hline Hospitalization in the last year & $144(19.5)$ & $205(25.5)$ & $<0.01$ \\
\hline $\begin{array}{l}\text { Antibiotherapy in the last } 3 \\
\text { months }\end{array}$ & $288(39.0)$ & $304(37.9)$ & 0.64 \\
\hline ESBL-E carrier at ICU admission & $34(4.6)$ & $35(4.4)$ & 0.09 \\
\hline $\begin{array}{l}\text { Diagnosis on admission to the ICUb } \\
\text { n (\%) }\end{array}$ & & & $<0.01$ \\
\hline Sepsis & $237(32.1)$ & $211(26.3)$ & \\
\hline Acute renal failure & $20(2.7)$ & $30(3.7)$ & \\
\hline Acute respiratory failure & $59(8.0)$ & $52(6.5)$ & \\
\hline Neurological failure & $115(15.6)$ & $98(12.2)$ & \\
\hline Polytrauma & $93(12.6)$ & $137(17.1)$ & \\
\hline Acute heart failure & $38(5.1)$ & $78(9.7)$ & \\
\hline Surgical emergency & $56(7.6)$ & $46(5.7)$ & \\
\hline Sickle cell disease & $11(1.5)$ & $25(3.1)$ & \\
\hline
\end{tabular}


a Obesity defined by body mass index $>30$

${ }^{\mathrm{b}}$ Main diagnosis made at admission

\# When not specified, results are $\mathrm{n}(\%)$. In bold, $p$ values $<0.05$

SAPS II: Simplified Acute Physiology Score II; ICU: intensive care unit; ESBL-E: extended-spectrum betalactamase-producing Enterobacteriacea e;SD: standard deviation

\section{Antibiotic Use}

During the RSP, the number of patients treated with antibiotics was significantly lower than during the CSP $(46.8 \%$ vs $57.9 \%$, respectively; $p<0.01)$. Median duration of antibiotic treatment was shorter by one day in the RSP ( 5 vs 6 days; $p<0.01$ ) (Table 2). Third-generation cephalosporins (ceftriaxone, cefotaxime) were the most commonly-administered antibiotics in both periods. More patients were treated with amoxicillin and cefuroxime in the RSP, and less patients received amoxicillin-clavulanate. Moreover, the administration of piperacillin/tazobactam and carbapenem was significantly reduced in the RSP (4.5\% vs $39.8 \%, p<0.01$ and $3.5 \%$ vs $12.5 \%, p<0.01$, respectively). Conversely there were more patients treated with ceftazidime, cefepime and cefoxitin in the RSP compared to the CSP. Importantly, the use of antibiotics targeting anaerobes pathogens (amoxicillin/clavulanic acid, piperacillin/tazobactam, carbapenem, metronidazole, cefoxitin and clindamycin) decreased in the RSP $(65.3 \%$ vs $33.5 \% ; p<0.01)$ and a large number of patients did not receive any antibiotic treatment during ICU stay.

Table 2. Sepsis events and antibiotherapy characteristics used in the intensive care unit during the study period. 
Sepsis events and antibiotherapy characteristics
Conventional strategy period
Restrictive strategy period

2015

$n=738$

$380(51.5)$ $n=803$

$327(40.7)$
2014 $p$ value
No. patients with at least one sepsis event $\mathbf{n}$

(\%)

(community or acquired)

Catecholamines administered for sepsis

No. patients receiving antibiotics $n(\%)$

No. of different antibiotics (median \pm IQR)

Duration of antibiotic therapy (days, median \pm IQR)

Antibiotic-free days until ICU discharge (days, $\quad 0$ [0-6] median \pm IQR)

Antibiotics administered $\mathbf{n}(\%)$

Amoxicillin

Amoxicillin/clavulanic acid

Oxacillin

Piperacillin/tazobactam

Cefazolin (C1G)

Cefuroxime (C2G)

Cefotaxime/ceftriaxone (C3G)

Cefoxitin (cephamycin)

Ceftazidime

Cefepim

Carbapenem

Vancomycin

Aminoglycoside

Fluoroquinolone

Macrolide
$29(6.8)$

$115(26.9)$

$20(4.7)$

$170(39.8)$

$1(0.2)$

$1(0.2)$

$192(45.0)$

$9(2.1)$

19 (4.4)

7 (1.6)

$52(12.2)$

$43(10.1)$

162 (37.9)

13 (3.0)

63 (14.8)
73 (19.4)

65 (17.3)

28 (7.4)

17 (4.5)

13 (3.5)

72 (19.1)

159 (42.3)

34 (9.0)

47 (12.5)

18 (4.8)

13 (3.5)

8 (2.1)

114 (30.3)

45 (12.0)

55 (14.6)
0.06 $<0.01$

0.55

$<0.01$

0.03 $<0.01$ 


\begin{tabular}{|cllc|}
\hline Clindamycin & $4(0.9)$ & $5(1.3)$ & 0.74 \\
\hline Metronidazole & $17(4.0)$ & $19(5.1)$ & 0.46 \\
\hline Trimethoprim/sulfamethoxazole & $17(4.0)$ & $26(6.9)$ & 0.06 \\
\hline $\begin{array}{l}\text { Antibiotics targeting anaerobic pathogens }{ }^{\text {a }} \mathbf{n} \\
(\%)\end{array}$ & $\mathbf{2 7 9 ( 6 5 . 3 )}$ & $\mathbf{1 2 6 ( 3 3 . 5 )}$ & $<0.01$ \\
\hline
\end{tabular}

${ }^{a}$ Amoxicillin /clavulanic acid, piperacillin / tazobactam, carbapenem, cefoxitin, clindamycin, metronidazole

: C1G: first-generation cephalosporin; C2G: second-generation cephalosporin; C3G: third-generation cephalosporin; IQR: interquartile range.

\# When not specified, results are $\mathrm{n}(\%)$. In bold, $p$ values $<0.05$

\section{ESBL-E Acquisition in the ICU}

The incidence of ICU-acquired ESBL-E was lower in the RSP compared to the CSP (12.1\% vs $19.0 \%$; $p<0.01$ ) (Table 3). The main microorganisms identified were Klebsiella pneumoniae (86.1\%), Enterobacter spp. (19.8\%) and Escherichia coli (3.8\%) during the study period. The incidence of ICU-acquired ESBL-E infection was lower in the RSP (8.3\% vs $5.1 \%$; $p=0.01$ ); ICU-acquired ESBL-E bacteremia did not differ between the study periods. No carbapenemase-producing Enterobacteriaceae were isolated during the study period.

The median time of being free of ESBL-E was 19 days ( $95 \% \mathrm{Cl}, 17-25$ days) in the whole cohort. In univariate analysis, the median time of being free of ESBL-E was significantly shorter in the CSP compared to the RSP with 18 days ( $95 \% \mathrm{Cl}, 16-21$ days) and 22 days ( $95 \% \mathrm{Cl}, 16-\mathrm{NA}$ days), respectively $(\mathrm{HR}, 0.675$ [95\% Cl, 0.521-0.875]; $p<0.01)$ (Figure 2). After propensity score analysis, RSP was significantly associated with the median time of being free of ESBL-E in the ICU as a protective factor (HR, 0.746 [95\% $\mathrm{Cl}, 0.575-0.968] ; p=0.02)$ by weighting or by adjustment (HR, $0.751[95 \% \mathrm{Cl}, 0.578-0.977] ; p=0.03)$ (Table 4).

A subgroup analysis of patients receiving antibiotic therapy during ICU stay showed similar results (Table 3, Figure 2 and Table 4).

Table 3. Secondary outcomes in the whole cohort and in the subgroup of patients receiving antibiotic therapy in the ICU. 
Conventional

In the whole cohort n (\%)

ICU-acquired ESBL-E ${ }^{\text {a }}$

Klebsiella pneumoniae ESBL

Escherichia coli ESBL

Enterobacter cloacae ESBL

Others

ESBL-E infections

ESBL-E bacteremia

Duration of mechanical ventilation, days (median \pm IQR) $\mathrm{n}=1023$

Relapse or recurrence of sepsis during ICU stay

All-cause ICU mortality

All-cause hospital mortality

ICU length of stay, days (median \pm IQR)

Hospital length of stay, days (median \pm IQR)

Patients who did not receive antibiotic therapy

Patients receiving antibiotic therapy in the ICU n (\%)

ICU-acquired ESBL-E

ESBL-E infections

ESBL-E bacteremia

Relapse or recurrence of sepsis during ICU stay

All-cause ICU mortality

All-cause hospital mortality

Antibiotic-free days until ICU discharge, days mean \pm SD strategy period

2014

Restrictive strategy period

$p$ value

2015

$\mathrm{n}=\mathbf{8 0 3}$

$97(12.1) \quad<0.01$

$81(83.5) \quad 0.34$

$5(5.2)$

0.49

$4(2.9)$

20 (20.6)

0.80

2 (1.4)

0 (0)

0.5

61 (8.3)

41 (5.1)

0.01

34 (4.6)

35 (4.4)

0.8

5 [3-11]

4 [3-9]

0.06

$178(24.4)$

135 (16.8)

$<0.01$

211 (28.6)

181 (22.5)

$<0.01$

253 (34.3)

222 (27.6)

$<0.01$

6 [4-12]

5 [4-10]

$<0.01$

14 [7-27]

13 [6.5-23]

0.04

311 (42.1)

427 (53.2)

$<0.01$

$n=427$

$n=\mathbf{3 7 6}$

$<0.01$

126 (29.5)

80 (21.3)

$<0.01$

60 (14.1)

38 (10.1)

0.09

34 (8.0)

32 (8.5)

0.78

176 (41.9)

132 (35.2)

0.05

148 (34.7)

105 (27.9)

0.04

246 (57.6)

255 (67.8)

$<0.01$

$4.8 \pm 9.5$

$5,7 \pm 9.6$

0.03 


\begin{tabular}{|llll} 
All patients ICU length of stay $<\mathbf{4 8}$ hours $(\%)$ & $\mathbf{n = 2 4 2}$ & $\mathbf{n = 2 4 1}$ & 0.3 \\
\hline All-cause ICU mortality & $65(26.9)$ & $66(27.4)$ & 0.9 \\
\hline All-cause hospital mortality & $76(31.4)$ & $71(29.5)$ & 0.6 \\
\hline
\end{tabular}

ICU: intensive care unit; ESBL-E: extended-spectrum beta-lactamase-producing Enterobacteriaceae; SD: standard deviation; IQR: interquartile range.

a Several patients acquired more than one ESBL species. This explains why the cumulative proportions of each group of patients with ESBL species is over $100 \%$.

\# When not specified, results are $\mathrm{n}(\%)$. In bold, $p$ values $<0.05$

Table 4 Multivariate analysis associated with the median time of being free of ESBL-E.

\begin{tabular}{|c|c|c|c|c|c|c|}
\hline Variable & $\begin{array}{l}\text { Univariate } \\
\text { analysis } \\
\mathrm{HR}(95 \% \\
\mathrm{Cl})\end{array}$ & $\begin{array}{l}p \\
\text { value }\end{array}$ & \multicolumn{2}{|l|}{$\mathrm{HR}(95 \% \mathrm{Cl})$} & $\begin{array}{l}\text { Multivariate PPS } \\
\text { adjusted } \\
\text { analysis } \\
\text { HR }(95 \% \mathrm{Cl})\end{array}$ & $\begin{array}{l}p \\
\text { value }\end{array}$ \\
\hline \multicolumn{7}{|l|}{$\begin{array}{l}\text { In the whole } \\
\text { cohort }\end{array}$} \\
\hline \multirow{2}{*}{$\begin{array}{l}\text { Restrictive } \\
\text { strategy (2015) }\end{array}$} & 0.675 & \multirow[t]{2}{*}{$<0.01$} & 0.746 & \multirow[t]{2}{*}{0.02} & 0.751 & \multirow[t]{2}{*}{0.03} \\
\hline & $\begin{array}{l}(0.521- \\
0.875)\end{array}$ & & $(0.575-0.968)$ & & $(0.578-0.977)$ & \\
\hline \multicolumn{7}{|c|}{$\begin{array}{l}\text { Patients receiving } \\
\text { antibiotherapy }\end{array}$} \\
\hline \multirow{2}{*}{$\begin{array}{l}\text { Restrictive } \\
\text { strategy (2015) }\end{array}$} & 0.712 & \multirow[t]{2}{*}{0.01} & 0.734 & \multirow[t]{2}{*}{0.03} & 0.738 & \multirow[t]{2}{*}{0.03} \\
\hline & $\begin{array}{l}(0.538- \\
0.941)\end{array}$ & & $(0.555-0.972)$ & & $(0.556-0.980)$ & \\
\hline
\end{tabular}

ICU: intensive care unit; $\mathrm{Cl}$, confidence interval; HR: hazard ratio; ESBL-E: extended-spectrum betalactamase-producing Enterobacteriaceae; PPS: propensity score

*Statistical significance level $<0.05$. All those variables were included to calculate the propensity score (age, SAPS II, chronic renal failure, diabetes, immunosuppression, antibiotherapy prior to admission, sepsis [at admission or occurred during ICU stay], other multidrug bacteria carriage at admission, mechanical ventilation duration $>48 \mathrm{~h}$ )

\section{Secondary Outcomes}


All-cause ICU mortality were lower in the RSP than in the CSP $(22.5 \%$ vs $28.6 \% ; p<0.01)$, including in the subgroup of patients receiving antibiotic therapy. Similar ICU mortality was observed in patients admitted for a length of stay $<48$ hours as safety criteria (Table 3 ).

\section{Discussion}

This study is the first to describe the effect of a very restrictive antibiotic stewardship program in ICU, with a particular emphasis on the impact on ESBL-E acquisition. An important finding was a reduction in ESBL-E acquisition associated to the antibiotic stewardship program with no excess in mortality rate, in the context of high prevalence in an endemic region, such as the French West Indies (18). This finding is consistent with previous studies showing a significant reduction of the incidence of antibiotic-resistant bacteria, including in ICU setting, following the implementation of antibiotic stewardship programs (19, 20). The lower acquisition rate in our ICU was associated with a significant reduction of the use of broadspectrum antibiotics, mainly piperacillin/tazobactam and carbapenem and, more generally, antibiotics targeting anaerobic microbiota. Several authors have reported the role of antibiotic therapies with activity against anaerobic microbiota, as well as the beta-lactamase inhibitor, in the acquisition of multidrugresistant Gram-negative bacteria, including $\operatorname{ESBL-E}(5,21)$. Furthermore, preserving the microbiota against antibiotics is one of the key strategies against ESBL-E acquisition (22).

The reluctance of ICU physicians to rationalize antibiotic use is often a major limitation in stewardship programs. We were able to overcome this unwillingness in our ICU, resulting in a significant reduction in global antibiotic consumption, including broad-spectrum antibiotics, a shorter duration of therapy, and an increased use of narrow-spectrum molecules. Interestingly, we observed a dramatic decrease of the consumption of carbapenems in the RSP, even though the prevalence of ESBL-E was high. The precise cause of the lower incidence of ESBL-E acquisition in the RSP is difficult to determine, but the strong decrease in the use of piperacillin/tazobactam may have played a role, as well as the choice of alternative antibiotics, such as cefoxitin. In the RSP, antibiotic duration was reduced by one day, although the baseline duration was already short. This is consistent with the current trend of a shorter treatment duration for infections such as VAP (23), intra-abdominal infections $(24,25)$, or bacteremia $(26)$. Discontinuation of antibiotic therapy in the case of negative microbiological cultures could also explain the short treatment duration, especially in $\operatorname{VAP}(27,28)$. Antibiotic duration is frequently longer than recommended in clinical practice (29), in spite of no better outcome, and the likely promotion of bacterial or fungal superinfections (27). Indeed, Daneman and colleagues showed that up to two-thirds of ICU patients with bacteremia did not receive a short course as recommended (30).

Lower antibiotic consumption during the RSP was also the result of the use of a lower threshold for the initiation of antibiotic therapy (7). In the case of hemodynamically-stable patients with suspected infection, antibiotic therapy was initiated only after clinical evidence of infection and microbiological documentation. In some patients, an alternative diagnosis was identified and antibiotic therapy was not initiated. This "conservative" strategy has been evaluated by Hranjec and colleagues in critically-ill surgical patients and resulted in a higher appropriate initial antibiotic treatment, reduced duration of 
antibiotic treatment, and a lower mortality rate (10). Despite the contradiction of delaying the initiation of antimicrobial treatment with pre-existing dogma and the promoted de-escalation strategy, several arguments support this approach (31). First, a high proportion of patients with fever received antimicrobial therapy in the ICU, despite non-infectious disease (32). Similarly, overdiagnosis of VAP was reported in $68 \%$ of patients, resulting in an overuse of antimicrobial therapy (33). Second, the linear association between the timing of antibiotic therapy and mortality has been challenged (34), suggesting no benefit in an immediate start of antimicrobial drugs in less severely-ill patients. Third, this position has been recently advocated by many experts (35). Of note, the Infectious Diseases Society of America decided not to endorse the 2018 Surviving Sepsis Campaign experts highlighted its recommendation of prompt antibiotic therapy (36), which they regarded as "an oversimplified approach" that should be reconsidered and potentially delayed in less severely-ill patients to avoid "treating some infections inadequately and others excessively" (37).

Our study has some limitations. First, it is a single-center study. Nevertheless, our results regarding the implementation of the stewardship program and its feasibility could be extrapolated to other ICUs. Second, outcomes related to the restrictive strategy cannot be addressed accurately as patients in the RSP were less severe, based on SAPS II at admission. However, SAPS II were included in the propensity score analysis. Furthermore, the standardized mortality ratio (ratio of actual ICU-mortality to SAPS IIpredicted mortality) was similar (0.93 and 0.95 , respectively) in both periods, suggesting no excess in the RSP mortality rate. Third, infection may have been overdiagnosed during the CSP, where the diagnosis was based on the physician's judgment. As previously mentioned, the diagnosis of infection may be difficult in the ICU and many non-infectious diseases may present with fever or inflammatory syndrome, especially when it comes to VAP (33). Conversely, during the RSP, a microbiological confirmation and/or a severity signs of sepsis were required to define infection. In the data collection process, those situations were called "infection", probably leading to some falsely reported infections during the CSP. As a suspicion of infection was often the trigger for initiating an empirical antibiotic therapy during the CSP, it is likely that the overdiagnosis of infection has played a role in the broader use of antibiotics, compared to the RSP in which a microbiological confirmation of infection and/or a severity sign were required to initiate empirical antibiotics. Neverless, the proportion of patients treated with vasoactive drugs for septic shock was similar, suggesting no difference in the incidence of the most severe patients between groups. Fourth, the rate of initial appropriate antibiotic therapy in septic shock was not reported. The use of cefoxitin as empirical treatment for acquired non-pulmonary septic shock was based on our local epidemiology, i.e., a high prevalence of cefoxitin susceptibility to ESBL-E, low incidence of AmpChyperproducing Enterobacteriaceae, and a very rare incidence of carbapenemase-producing Enterobacteriaceae and MRSA. Although administration of cefoxitin was appropriate when referring to antimicrobial susceptibility testing, few data support its clinical use in severe infections, other than urinary tract infection (38). To address this issue, we used a combination therapy of cefoxitin and amikacin for empirical treatment in acquired septic shock.

\section{Conclusions}


A restrictive strategy delaying the initiation of antibiotic treatment in less severely-ill patients and using the narrowest spectrum has the potential to avoid broad-spectrum antibiotics, particularly those targeting intestinal anaerobe microbiota. This strategy resulted in a dramatic decrease in antibiotic consumption and was independently associated with a reduction of ESBL-E acquisition in the ICU.

\section{Abbreviations}

ESBLE-E: extended-spectrum beta-lactamase-producing Enterobacteriaceae; ICU: Intensive care unit; CSP: conventional strategy period; RSP; restrictive strategy period; ARDS: acute respiratory distress syndrome; MRSA: methicillin-resistant Staphylococcus aureus; VAP: Ventilatory-associated pneumonia; SAPS II: Simplified Acute Physiology Score II

\section{Declarations}

Acknowledgement: We sincerely thank Rosemary Sudan for the proofreading of this manuscript.

Authors' contributions: CLT, MV and GT conceived and designed the study. They had full access to all data in the study and take responsibility for the integrity of the data and the accuracy of the data analysis. CLT, MV and RR collected the data. CLT, PB, EO, MC and GT analysed the data. CLT, MV and GT drafted the paper. CLT and MV contributed equally to this work. All authors interpreted the data and critically revised the manuscript for important intellectual content and gave approval for the final version to be published. All authors agree to be accountable for all aspects of the work in ensuring that questions related to the accuracy or integrity of any part of the work are appropriately investigated and resolved.

The manuscript's guarantors (CLT, MV and GT) affirm that the manuscript is an honest, accurate, and transparent account of the study being reported; that no important aspects of the study have been omitted; and that any discrepancies from the study as planned have been explained.

Funding: No funding was received for this work.

Availability of data and materials: After publication, the data will be made available upon reasonable request to the corresponding author. A proposal with a detailed description of study objectives and statistical analysis plan will be needed for evaluation of the reasonability of requests. Additional materials might also be required during the process of evaluation. De-identified participant data will be provided after approval from the corresponding author and the Guadeloupe University Hospital, French West Indies.

\section{Declarations}

Ethics approval and consent to participate: The ethics committee of the French Society of Intensive Care Medicine (CE SRLF 18-44) approved the study and granted a waiver for informed consent because that both treatment methods were classified as standard care. 
Consent for publication: All authors have seen and approved the manuscript. Drs. Christophe Le Terrier and Marco Vinetti are co-first authors and have contributed equally to this work.

Competing interests: All authors declare no conflicts of interest.

\section{References}

1. WHO. Antimicrobial resistance: global report on surveillance. World Health Organization, Geneva, 2014. https://www.who.int/antimicrobial-resistance/publications/surveillancereport/en/: accessed May 28, 2020.

2. WHO. Global priority list of antibiotic-resistant bacteria to guide research, discovery, and development of new antibiotics. World Health Organization, Geneva, 2017.

https://www.who.int/medicines/publications/global-priority-list-antibiotic-resistant-bacteria/en/: accessed May 19, 2020.

3. Zahar JR, Blot S, Nordmann P, et al. Screening for Intestinal Carriage of Extended-spectrum Betalactamase-producing Enterobacteriaceae in Critically III Patients: Expected Benefits and Evidencebased Controversies. Clin Infect Dis 2019; 30: 2125-2130.

4. Ruppé $\mathrm{E}$, Burdet $\mathrm{C}$, Grall $\mathrm{N}$, et al. Impact of antibiotics on the intestinal microbiota needs to be redefined to optimize antibiotic usage. Clin Microbiol Infect 2018; 24: 3-5.

5. Razazi K, Derde LPG, Verachten M, Legrand P, Lesprit P, Brun-Buisson C. Clinical impact and risk factors for colonization with extended-spectrum $\beta$-lactamase-producing bacteria in the intensive care unit. Intensive Care Med 2012; 38: 1769-1778.

6. Versporten A, Zarb P, Caniaux I, et al. Antimicrobial consumption and resistance in adult hospital inpatients in 53 countries: results of an internet-based global point prevalence survey. Lancet Glob Health 2018; 6: e619-629.

7. De Waele JJ, Akova M, Antonelli M, et al. Antimicrobial resistance and antibiotic stewardship programs in the ICU: insistence and persistence in the fight against resistance. A position statement from ESICM/ESCMID/WAAAR round table on multi-drug resistance. Intensive Care Med 2018; 44: 189-196.

8. De Waele JJ, Schouten J, Dimopoulos G. Understanding antibiotic stewardship for the critically- ill. Intensive Care Med 2016; 42: 2063-2065.

9. Timsit J-F, Bassetti M, Cremer O, et al. Rationalizing antimicrobial therapy in the ICU: a narrative review. Intensive Care Med 2019; 45: 172-189.

10. Hranjec T, Rosenberger LH, Swenson B, et al. Aggressive versus conservative initiation of antimicrobial treatment in critically ill surgical patients with suspected intensive-care-unit-acquired infection: A quasi-experimental, before and after observational cohort study. Lancet Infect Dis 2012; 12:774-780.

11. Chaintarli K, Lenglet A, Beauzile BD, et al. High prevalence of ESBL-positive bacteria in an obstetrics emergency hospital and neonatal care unit-Haiti. Infect Control Hosp Epidemiol 2018; 11: 1381-1383. 
12. Heinz E, Brindle R, Morgan-McCalla A, Peters K, Thomson NR. Caribbean multi-centre study of Klebsiella pneumoniae: whole-genome sequencing, antimicrobial resistance and virulence factors. Microb Genom 2019; 5: e000266.

13. Luna CM, Rodriguez-Noriega E, Bavestrello L, Guzmán-Blanco M. Gram-negative infections in adult intensive care units of latin america and the Caribbean. Crit Care Res Pract 2014; 2014: 480463.

14. Société française d'anesthésie et de réanimation, Société de réanimation de langue française. [Prevention of hospital-acquired sepsis in intensive care unit (except cross transmission and neonate)]. Ann Fr Anesth Reanim 2009;28:912-20.

15. Weiss $E$, Zahar JR, Lesprit $P$, et al. Elaboration of a consensual definition of de-escalation allowing a ranking of $\beta$-lactams. Clin Microbiol Infect 2015; 21:649.e1-10.

16. The European Committee on Antimicrobial Susceptibility Testing. Breakpoint tables for interpretation of MICs and zone diameters. Version 11.0, 2021. http://www.eucast.org

17. Chastre J, Luyt C-E. Does this patient have VAP? Intensive Care Med 2016; 42: 1159-1163.

18. Quiñones D, Valverde A, Rodríguez-Baños $M$, et al. High clonal diversity in a non-outbreak situation of clinical ESBL-producing Klebsiella pneumoniae isolates in the first national surveillance program in Cuba. Microb Drug Resist 2014; 20: 45-51.

19. Baur D, Gladstone BP, Burkert F, et al. Effect of antibiotic stewardship on the incidence of infection and colonisation with antibiotic-resistant bacteria and Clostridium difficile infection: a systematic review and meta-analysis. Lancet Infect Dis 2017; 17: 990-1001.

20. Teerawattanapong N, Kengkla K, Dilokthornsakul P, Saokaew S, Apisarnthanarak A, Chaiyakunapruk N. Prevention and control of multidrug-resistant gram-negative bacteria in adult intensive care units: A systematic review and network meta-analysis. Clin Infect Dis 2017; 64: S51-60.

21. Harris AD, McGregor JC, Johnson JA, et al. Risk factors for colonization with extended-spectrum $\beta$ lactamase- producing bacteria and intensive care unit admission. Emerg Infect Dis 2007; 13: 11441149.

22. Bassetti M, Poulakou G, Ruppe E, Bouza E, Van Hal SJ, Brink A. Antimicrobial resistance in the next 30 years, humankind, bugs and drugs: a visionary approach. Intensive Care Med 2017;43:14641475.

23. Klompas M, Li L, Menchaca JT, Gruber S. Ultra short course antibiotics for patients with suspected ventilator-associated pneumonia but minimal and stable ventilator settings. Clin Infect Dis 2016; 64: ciw870.

24. Sawyer RG, Claridge JA, Nathens AB, et al. Trial of short-course antimicrobial therapy for intraabdominal infection. N Engl J Med 2015; 72: 1996.

25. Montravers $P$, Tubach F, Lescot $T$, et al. Short-course antibiotic therapy for critically ill patients treated for postoperative intra-abdominal infection: the DURAPOP randomised clinical trial. Intensive Care Med 2018; 44: 300-310. 
26. Havey TC, Fowler RA, Daneman N. Duration of antibiotic therapy for bacteremia: a systematic review and meta-analysis. Crit Care 2011; 15: R267

27. Raman K, Nailor MD, Nicolau DP, Aslanzadeh J, Nadeau M, Kuti JL. Early antibiotic discontinuation in patients with clinically suspected ventilator-associated pneumonia and negative quantitative bronchoscopy cultures. Crit Care Med 2013; 41:1656-1663.

28. Kollef MH, Shorr A, Tabak YP, Gupta V, Liu LZ, Johannes RS. Epidemiology and outcomes of healthcare-associated pneumonia. Chest 2005; 128: 3854-3862.

29. De Waele JJ, Schouten J, Beovic B, et al. Antimicrobial de-escalation as part of antimicrobial stewardship in intensive care: no simple answers to simple questions-a viewpoint of experts. Intensive Care Med 2020; 46: 236-244.

30. Daneman N, Rishu AH, Xiong W, et al. Duration of antimicrobial treatment for bacteremia in canadian critically ill patients. Crit Care Med 2016; 44: 256-264.

31. Bernhard M, Lichtenstern C, Eckmann C, Weigand MA. The early antibiotic therapy in septic patients milestone or sticking point? Crit Care 2014; 18: 1-5.

32. Levin PD, Idrees S, Sprung CL, et al. Antimicrobial use in the ICU: indications and accuracy-an observational trial. $J$ Hosp Med 2012; 7: 672-678.

33. Nussenblatt V, Avdic E, Berenholtz S, et al. Ventilator-associated pneumonia: overdiagnosis and treatment are common in medical and surgical intensive care units. Infect Control Hosp Epidemiol 2014;35: 278-284.

34. Sterling SA, Miller WR, Pryor J, Puskarich MA, Jones AE. The impact of timing of antibiotics on outcomes in severe sepsis and septic shock: a systematic review and meta-analysis. Crit Care Med 2015;43: 1907-1915.

35. Da Fonseca Pestana Ribeiro JM, Park M. Less empiric broad-spectrum antibiotics is more in the ICU. Intensive Care Med 2020;46: 783-786.

36. Rhodes A, Evans LE, Alhazzani W, et al. Surviving Sepsis Campaign: international guidelines for management of sepsis and septic shock: 2016. Intensive Care Med 2017;43: 304-377.

37. Kalil AC, Gilbert DN, Winslow DL, Masur H, Klompas M. Infectious Diseases Society of America (IDSA) position statement: Why IDSA did not endorse the surviving sepsis campaign guidelines. Clin Infect Dis 2018;66: 1631-1635.

38. Kernéis S, Valade S, Geri G, et al. Cefoxitin as a carbapenem-sparing antibiotic for infections caused by extended-spectrum beta-lactamase-producing Escherichia coli and Klebsiella pneumoniae. Infect Dis 2015;47: 789-795.

\section{Figures}




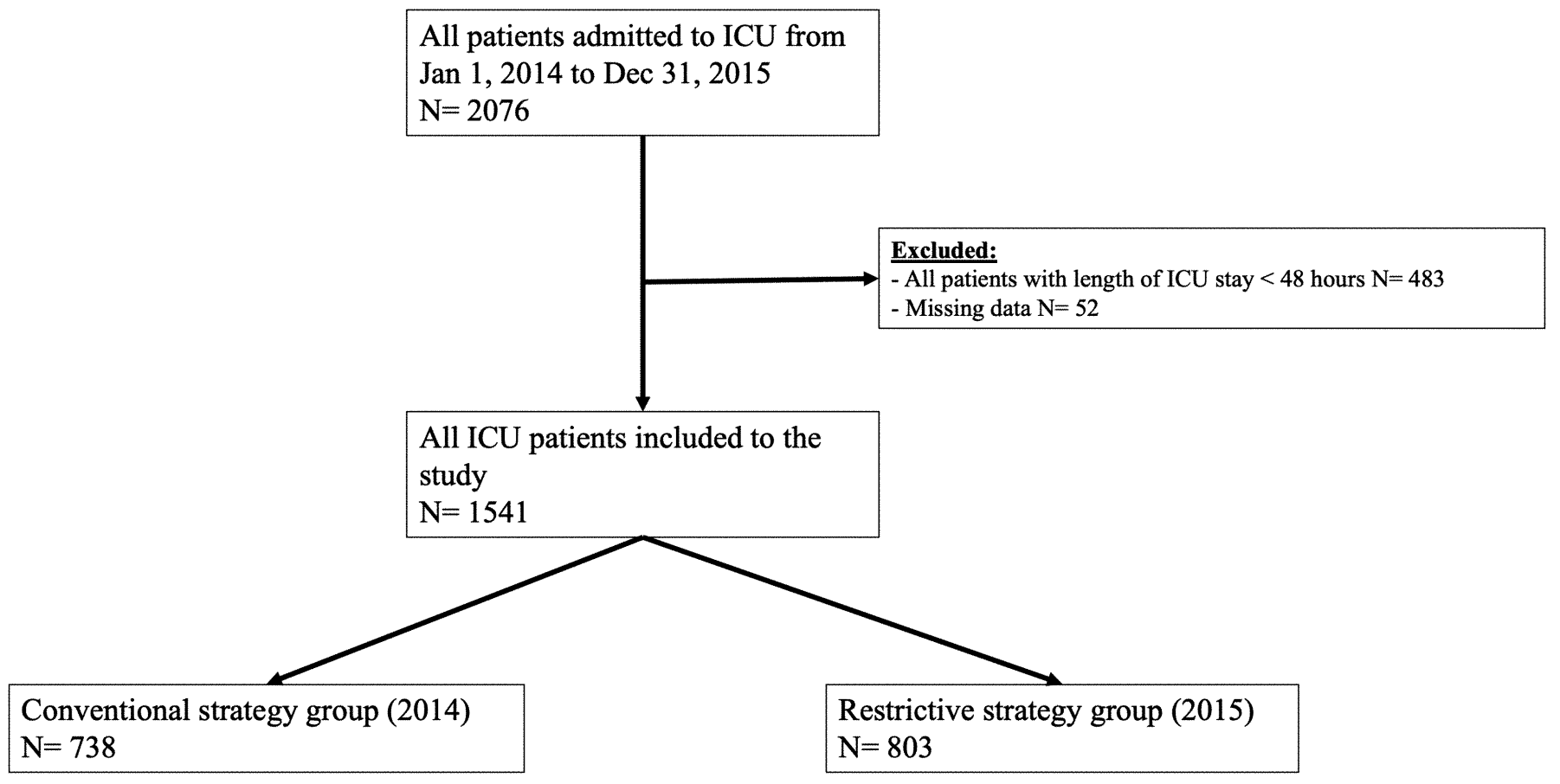

\section{Figure 1}

Study flow chart. ICU: intensive care unit 

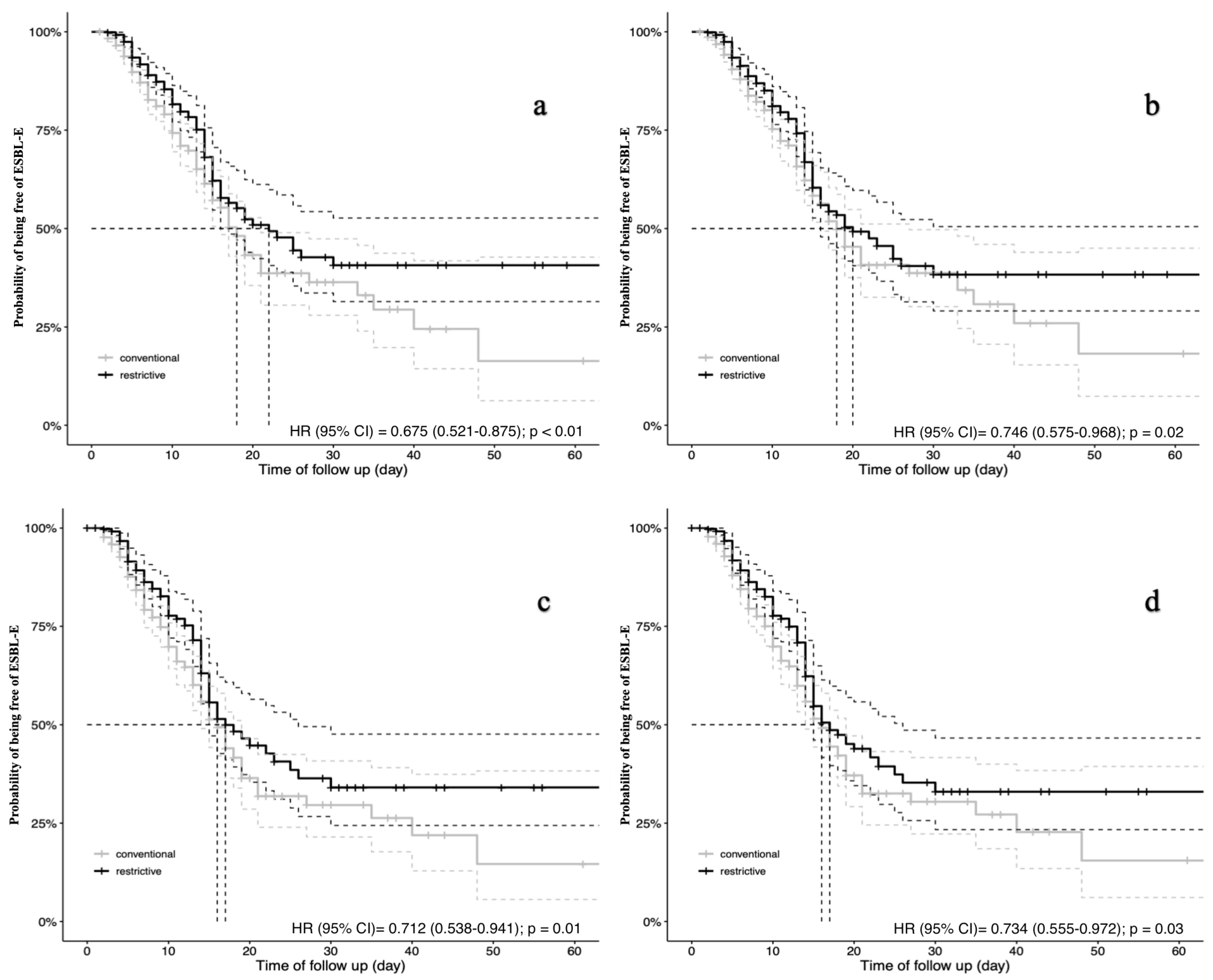

Figure 2

Kaplan-Meier curves for the probability of being free of ESBL-E. a) Unweighted Kaplan-Meier survival curves by the strategy used in the all-ICU patients. b) Propensity score-weighted Kaplan-Meier survival curves by the strategy used in the all-ICU patients. c) Unweighted Kaplan-Meier survival curves by the strategy used in the subgroup of patients receiving antibiotherapy. d) Propensity score-weighted KaplanMeier survival curves by the strategy used in the subgroup of patients receiving antibiotherapy. ESBL-E: extended-spectrum beta-lactamase-producing Enterobacteriaceae HR: hazard ratio

\section{Supplementary Files}

This is a list of supplementary files associated with this preprint. Click to download.

- AdditionalFile.pdf 